\title{
Métodos de ADN Barcode revelan diversidad de especies de Astroblepus en la cuenca del río Porce, Colombia
}

\author{
DNA Barcode methods reveal diversity of Astroblepus species in the Porce River \\ drainage, Colombia
}

\author{
Marcela Hernández-Zapata ${ }^{1 *}$, Luz E. Ochoa-Orrego², Luz F. Jiménez-Segura1
}

\begin{abstract}
Resumen
El género Astroblepus es endémico de la región Andina y está representado por aproximadamente 81 especies válidas que habitan ríos de alta montaña desde Panamá hasta Bolivia. Para Colombia, se han registrado 32 especies, de las cuales 23 fueron registradas en monitoreos recientes en la cuenca del río Porce, indicando que cerca del $71 \%$ de la diversidad de especies se encuentra en esta área. Morfológicamente, las especies de Astroblepus son muy similares y los caracteres empleados tradicionalmente para su determinación son poco resolutivos, lo que dificulta su identificación taxonómica. Con el objetivo de determinar la diversidad de Astroblepus en la cuenca del río Porce, se analizaron 129 individuos empleando herramientas de identificación genética mediante la secuenciación del gen Citrocromo Oxidasa sub-unidad I (COI) de $580 \mathrm{pb}$ y un análisis morfométrico basado en proporciones corporales, usando 12 hitos morfológicos. Los análisis genéticos y el método de delimitación de especies "General mixed Yule-coalescent", indicaron la presencia de 12 entidades taxonómicas, las cuales fueron soportadas por sus distancias genéticas y por 13 medidas corporales basadas en proporciones. Los resultados indican que la diversidad de especies en el área ha sido sobreestimada, por lo que es necesario integrar diferentes metodologías para una correcta delimitación de especies.
\end{abstract}

Palabras claves: ADN mitocondrial, gen COI, GMYC, ictiofauna neotropical, distancias genéticas, proporciones

\begin{abstract}
The genus Astroblepus, is endemic to the Andean region and is represented by 81 valid species inhabiting high mountain rivers from Panama to Bolivia. In Colombia, about 32 species of this genus have been reported, of which 23 were recorded in the Porce River drainage, which indicates that about $71 \%$ of species diversity can be found in this area. Morphologically the species of Astroblepus are very similar and the characters traditionally used for their determination are not very diagnostic, which limits their identification at species level. In order to determine the diversity of Astroblepus in the Porce River drainage, 129 individuals were analyzed using genetic identification tools through the sequencing of the $580 \mathrm{bp}$ Cytochrome Oxidase subunit I ( $\mathrm{COI}$ ) gene and an analysis based on morphometry. The genetic analyses and the method of delimitation of species "General mixed Yule-coalescent", indicated the presence of 12 taxonomic entities, which were supported by genetic distances and by 13 body measurements based on corporal proportions. The results indicate that the diversity of species has been overestimated in this river and highlight the importance to integrate different methodologies in the delimitation species studies.
\end{abstract}

Key words: mitochondrial DNA, COI gene, GMYC, Neotropical ichthyofauna, genetic distances, morphometrics

1. Grupo de Ictiología Universidad de Antioquia, Medellín, Colombia.

2. Laboratorio de Biología e Genética de Peixes, Departamento de Morfología, Universidad Estadual Paulista-UNESP, São Paulo, Brasil.

* Autor para correspondencia: <marcehz9@hotmail.com>

Recibido: junio de 2017; aceptado: abril de 2018. 


\section{INTRODUCCIÓN}

La familia Astroblepidae es un grupo monotípico, representado por el género Astroblepus, Humboldt, 1805, con 81 especies válidas (Eschmeyer y Fong 2017), registrando para Colombia 32 especies, por lo que es considerado el país con el mayor número de especies de esta familia (Schaefer 2003). Este grupo, endémico del Neotrópico, se encuentra distribuido desde Panamá hasta Bolivia a $28^{\circ}$ de latitud sur aproximadamente (Schaefer et al. 2011), ocurriendo principalmente en ambientes correntosos.

Morfológicamente, presentan varias modificaciones que les permiten distribuirse en dichos ambientes, como la boca en forma de ventosa y aletas pélvicas móviles (Johnson 1912) que les permiten desplazarse corriente arriba, razón por la cual se les ha llamado comúnmente como "escaladores". Generalmente, las especies del género habitan sistemas altamente conservados, con aguas limpias y oxigenadas, soportando altas velocidades en el flujo del agua $(>130 \mathrm{~cm} / \mathrm{s})$ (Miranda-Chumacero 2006). Poseen una distribución altitudinal que se extiende entre los 100 y 4600 m s.n.m., con mayor frecuencia entre los 500 y 2000 m s.n.m. (Schaefer y Arroyave 2010). Las especies de esta familia toleran bajas temperaturas del agua, lo que les ha permitido la colonización y el establecimiento en ambientes de alta montaña (Jacobsen 2008, Miles 1971) y actualmente son considerados un importante bioindicador del estado de conservación de los sistemas en que habitan (Schaefer et al. 2011).

Tal como fue planteado por Schaefer et al. (2011), la identificación morfológica de las especies de Astroblepus no es una tarea fácil, debido a que la mayoría son definidas por un único carácter contrastante o por combinaciones de caracteres externos que presentan altos niveles de variación inter e intraespecífica, llevando en muchas ocasiones a la sobreposición entre especies. Después del trabajo de Regan (1904), no existe, hasta el momento, una revisión taxonómica integral de las especies del género que permita establecer la identidad taxonómica de las especies presentes en Colombia. Muchas de las especies identificadas en listados regionales y nacionales, presentan epítetos de especies descritas originalmente para regiones distantes de otros países; como ejemplo, Astroblepus longifilis (Steindachner, 1882) del Amazonas ecuatoriano, ha sido registrada para las cuencas trasandinas de los ríos Magdalena y Cauca y ríos de la vertiente Pacífica de Colombia. Así mismo, especies como A. homodon (Regan, 1904) y A. longifilis, aparentemente presentan una amplia distribución en el territorio nacional, situación que es necesario evaluar considerando el alto grado de endemismo del grupo.

Un claro ejemplo de esta situación fue identificado en estudios de composición y estructura de la comunidad íctica realizados entre los años 2005 y 2012 para la cuenca del río Porce, donde fueron registradas nueve especies: A. trifasciatus (Eigenmann, 1912), A. unifasciatus (Eigenmann, 1912), A. chapmani (Eigenmann, 1912), A. guentheri (Boulenger, 1887), A. grixalvii Humboldt 1815, A. supramollis Pearson, 1937, A. cyclopus (Humboldt, 1805), A. latidens Eigenmann, 1918 y A. micrescens Eigenmann, 1918, en cuanto varios individuos solo fueron identificadas a nivel de género "sp", y otros fueron reconocidos en las categorías "cf" (comparar con) y "aff" (afin a). De este conjunto, algunas especies tienen localidades tipo en otros países como $A$. supramollis descrita para la localidad de las Balsas en Perú y $A$. cyclopus descrita para el río Esmeraldas en Ecuador; así mismo, dentro de las especies registradas para Colombia, algunas de las especies identificadas en el río Porce tienen localidades en regiones distantes como $A$. trifasciatus y A. unifasciatus descritas para el río Dagua, A. chapmani descrita para la quebrada Boquia en Quindío, A. latidens descrita para la localidad Piperel y distribuida en la cuenca alta del río Meta y $A$. micrescens, descrita de la quebrada de Agua Larga al norte de Bogotá.

Para intentar identificar las especies distribuidas en la cuenca del río Porce, Hernández et al. (2016) realizaron un análisis de morfometría geométrica usando 12 puntos homólogos de tipo I, II y III; sin embargo, los resultados no fueron estadísticamente significativos $(p=0,5194)$ y no fue posible observar una tendencia de agrupamiento de los datos que correspondiera con la identificación previa de las especies.

Considerando las dificultades en la identificación morfológica de este grupo de especies y la necesidad de esclarecer su identidad taxonómica para monitorear el ensamblaje de sus especies en la región, empleamos la secuencia del gen citocromo oxidasa I (COI) para la identificación genética de los individuos de acuerdo con la metodología propuesta por Hebert et al. 2003; adicionalmente, identificamos caracteres fenotípicos para cada una de las unidades genéticas observadas, principalmente basados en proporciones morfológicas. De acuerdo con los resultados obtenidos proponemos una clave para la identificación de especies del género presentes en la cuenca del río Porce. 


\section{MATERIALES Y MÉTODOS}

Área de estudio. El río Porce atraviesa el Valle de Aburrá como extensión del río Medellín, iniciando su curso en el alto de San Miguel a 2660 m s.n.m. atravesando la parte central del departamento de Antioquia en dirección noreste (EPM 2004, Jiménez-Segura et al. 2014). Su valle es un cañón profundo en forma de "V" con pendientes pronunciadas y asimétricas normalmente mayores del $50 \%$. La temperatura de esta región es de aproximadamente 21 ${ }^{\circ} \mathrm{C}$ con una precipitación anual de $2248 \mathrm{~mm}$. Los sistemas de primer orden o quebradas afluentes se caracterizan por presentar alta transparencia del agua, elevadas velocidades en el flujo del agua, buena oxigenación, sustrato rocoso, vegetación lateral arbustiva y arbórea, los cuales, en conjunto, constituyen el hábitat de especies del género Astroblepus.

Recolecta de especímenes. Los especímenes fueron capturados con el uso de atarrayas y electropesca en 12 quebradas tributarias al río Porce, cuenca MagdalenaCauca (figura 1). Los ejemplares voucher fueron ingresados a la Colección de Ictiología de la Universidad de Antioquia (CIUA) e identificados previamente (tabla 1). Se obtuvo una muestra de tejido muscular de la base de la aleta dorsal de los individuos, la cual fue preservada en alcohol al 96\% y conservada a $-20{ }^{\circ} \mathrm{C}$.

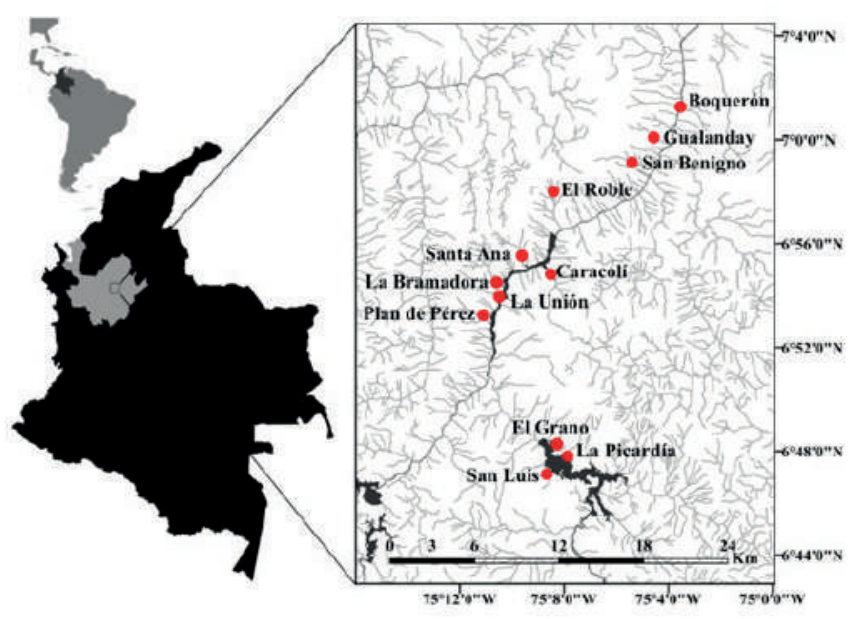

Figura 1. Ubicación geográfica de las 12 quebradas afluentes del río Porce en las cuales fueron capturados los ejemplares de Astroblepus.
Tabla 1. Identificación original de los individuos del género Astroblepus empleados en el análisis, su respectivo voucher, lote de colección y la clasificación mediante GYMC (OUT's)

\begin{tabular}{|c|c|c|c|}
\hline Identificación Previa & Voucher & Lote & OUT's \\
\hline Astroblepus grixalvii & 7442 & 1944 & $E$ \\
\hline Astroblepus trifasciatus & 7474 & 2020 & $\mathrm{H}$ \\
\hline Astroblepus supramollis & 7471 & 2205 & C \\
\hline Astroblepus unifasciatus & 7464 & 2206 & C \\
\hline Astroblepus trifasciatus & 7460 & 2207 & C \\
\hline Astroblepus unifasciatus & 7457 & \multirow{2}{*}{2208} & C \\
\hline Astroblepus unifasciatus & 7458 & & C \\
\hline Astroblepus micrescens & 7462 & 2210 & C \\
\hline Astroblepus chapmani & 7475 & 2211 & A \\
\hline Astroblepus supramollis & 7466 & 2212 & C \\
\hline Astroblepus sp. 1 & 7463 & 2214 & A \\
\hline Astroblepus trifasciatus & 7465 & 2215 & C \\
\hline Astroblepus cf grixalvii & 7480 & 2218 & $\mathrm{H}$ \\
\hline Astroblepus grixalvii & 7477 & 2226 & $\mathrm{H}$ \\
\hline Astroblepus chotae & 7476 & 2228 & $\mathrm{E}$ \\
\hline Astroblepus supramollis & 7469 & 2229 & C \\
\hline Astroblepus chotae & 7468 & 2230 & $\mathrm{E}$ \\
\hline Astroblepus aff latidens & 7461 & 2233 & C \\
\hline Astroblepus trifasciatus & 7472 & 2236 & C \\
\hline Astroblepus sp. 1 & 7481 & 2237 & A \\
\hline Astroblepus supramollis & 7528 & 2413 & $\mathrm{C}$ \\
\hline Astroblepus grixalvii & 7534 & 2415 & $E$ \\
\hline Astroblepus unifasciatus & 7530 & 2416 & C \\
\hline Astroblepus cyclopus & 7520 & 2417 & A \\
\hline Astroblepus trifasciatus & 7506 & 2419 & C \\
\hline Astroblepus chapmani & 7508 & \multirow{3}{*}{2421} & A \\
\hline Astroblepus chapmani & 7510 & & A \\
\hline Astroblepus chapmani & 7509 & & A \\
\hline Astroblepus guentheri & 7517 & 2422 & $\mathrm{~F}$ \\
\hline Astroblepus supramollis & 7515 & 2425 & C \\
\hline Astroblepus latidens & 7531 & 2426 & A \\
\hline Astroblepus unifasciatus & 7523 & 2429 & C \\
\hline Astroblepus trifasciatus & 7513 & 2432 & C \\
\hline Astroblepus sp. 3 & 7524 & 2434 & $\mathrm{H}$ \\
\hline Astroblepus supramollis & 7516 & 2435 & C \\
\hline Astroblepus cf latidens & 7522 & \multirow{2}{*}{2438} & A \\
\hline Astroblepus cf latidens & 7521 & & A \\
\hline Astroblepus trifasciatus & 7525 & \multirow{2}{*}{2441} & C \\
\hline Astroblepus trifasciatus & 7526 & & C \\
\hline Astroblepus trifasciatus & 7497 & 2450 & C \\
\hline Astroblepus grixalvii & 7565 & \multirow{2}{*}{2542} & $\mathrm{E}$ \\
\hline Astroblepus grixalvii & 7564 & & $\mathrm{C}$ \\
\hline Astroblepus sp. & 7573 & \multirow{2}{*}{2548} & $A$ \\
\hline Astroblepus sp. & 7572 & & $A$ \\
\hline Astroblepus sp.3 & 7575 & 2549 & $\mathrm{H}$ \\
\hline Astroblepus cf latidens & 7576 & 2550 & $\mathrm{H}$ \\
\hline Astroblepus unifasciatus & 7577 & \multirow{2}{*}{2551} & C \\
\hline Astroblepus unifasciatus & 7578 & & c \\
\hline
\end{tabular}




\begin{tabular}{|c|c|c|c|}
\hline Identificación Previa & Voucher & Lote & OUT's \\
\hline Astroblepus trifasciatus & 7579 & 2552 & $\mathrm{C}$ \\
\hline Astroblepus trifasciatus & 7581 & \multirow{2}{*}{2553} & C \\
\hline Astroblepus trifasciatus & 7582 & & C \\
\hline Astroblepus unifasciatus & 7597 & 2610 & A \\
\hline Astroblepus unifasciatus & 7600 & 2613 & C \\
\hline Astroblepus sp. 5 & 7967 & 3445 & C \\
\hline Astroblepus trifasciatus & 7944 & \multirow{2}{*}{3448} & C \\
\hline Astroblepus trifasciatus & 7945 & & C \\
\hline Astroblepus trifasciatus & 8000 & \multirow{2}{*}{3449} & C \\
\hline Astroblepus trifasciatus & 7999 & & C \\
\hline Astroblepus trifasciatus & 7965 & \multirow{2}{*}{3450} & C \\
\hline Astroblepus trifasciatus & 7968 & & $C$ \\
\hline Astroblepus trifasciatus & 7975 & \multirow{7}{*}{3451} & C \\
\hline Astroblepus trifasciatus & 7976 & & L \\
\hline Astroblepus trifasciatus & 7974 & & $\mathrm{~J}$ \\
\hline Astroblepus trifasciatus & 7969 & & C \\
\hline Astroblepus trifasciatus & 7978 & & C \\
\hline Astroblepus trifasciatus & 7973 & & $\mathrm{C}$ \\
\hline Astroblepus trifasciatus & 7977 & & C \\
\hline Astroblepus grixalvii & 7998 & \multirow{3}{*}{3453} & $\mathrm{E}$ \\
\hline Astroblepus grixalvii & 7997 & & $\mathrm{E}$ \\
\hline Astroblepus grixalvii & 8029 & & $\mathrm{E}$ \\
\hline Astroblepus trifasciatus & 7961 & \multirow{4}{*}{3456} & C \\
\hline Astroblepus trifasciatus & 7964 & & C \\
\hline Astroblepus trifasciatus & 7960 & & C \\
\hline Astroblepus trifasciatus & 7959 & & C \\
\hline Astroblepus sp.5 & 7947 & \multirow{2}{*}{3457} & C \\
\hline Astroblepus sp.5 & 7948 & & C \\
\hline Astroblepus trifasciatus & 8007 & \multirow{16}{*}{3458} & C \\
\hline Astroblepus trifasciatus & 8019 & & C \\
\hline Astroblepus trifasciatus & 8004 & & $\mathrm{D}$ \\
\hline Astroblepus trifasciatus & 8022 & & $c$ \\
\hline Astroblepus trifasciatus & 8013 & & C \\
\hline Astroblepus trifasciatus & 8005 & & C \\
\hline Astroblepus trifasciatus & 8021 & & 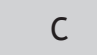 \\
\hline Astroblepus trifasciatus & 8017 & & $c$ \\
\hline Astroblepus trifasciatus & 8024 & & $\mathrm{C}$ \\
\hline Astroblepus trifasciatus & 8020 & & $c$ \\
\hline Astroblepus trifasciatus & 8016 & & C \\
\hline Astroblepus trifasciatus & 8018 & & C \\
\hline Astroblepus trifasciatus & 8008 & & C \\
\hline Astroblepus trifasciatus & 8014 & & C \\
\hline Astroblepus trifasciatus & 8023 & & C \\
\hline Astroblepus trifasciatus & 8003 & & $\mathrm{C}$ \\
\hline
\end{tabular}

\begin{tabular}{lccc}
\hline Identificación Previa & Voucher & Lote & OUT's \\
\hline Astroblepus chapmani & 7989 & 3460 & $\mathrm{~A}$ \\
\hline Astroblepus sp.5 & 7972 & & $\mathrm{I}$ \\
Astroblepus sp.5 & 7979 & 3466 & $\mathrm{C}$ \\
\hline Astroblepus guentheri & 7956 & & $\mathrm{C}$ \\
Astroblepus guentheri & 7957 & & $\mathrm{~A}$ \\
Astroblepus guentheri & 7958 & 3467 & $\mathrm{~A}$ \\
Astroblepus guentheri & 7955 & & $\mathrm{~K}$ \\
\hline Astroblepus sp.1 & 8030 & 3469 & $\mathrm{H}$ \\
\hline Astroblepus trifasciatus & 7949 & 3470 & $\mathrm{C}$ \\
\hline Astroblepus chapmani & 7971 & 3471 & $\mathrm{I}$ \\
\hline Astroblepus sp.1 & 7980 & 3475 & $\mathrm{C}$ \\
\hline Astroblepus trifasciatus & 7985 & & $\mathrm{C}$ \\
Astroblepus trifasciatus & 7992 & & $\mathrm{C}$ \\
Astroblepus trifasciatus & 7991 & & $\mathrm{C}$ \\
Astroblepus trifasciatus & 7993 & & $\mathrm{C}$ \\
Astroblepus trifasciatus & 7990 & & $\mathrm{C}$ \\
Astroblepus trifasciatus & 7986 & & $\mathrm{C}$ \\
\hline Astroblepus guentheri & 8119 & & $\mathrm{E}$ \\
Astroblepus guentheri & 8120 & & $\mathrm{E}$ \\
Astroblepus guentheri & 8121 & 3491 & $\mathrm{E}$ \\
Astroblepus guentheri & 8123 & & $\mathrm{G}$ \\
Astroblepus guentheri & 8114 & & $\mathrm{G}$ \\
Astroblepus cf unifasciatus & 7923 & & $\mathrm{C}$ \\
\hline Astroblepus guentheri & 8113 & & $\mathrm{G}$ \\
\hline Astroblepus cf unifasciatus & 7922 & 4225 & $\mathrm{C}$ \\
Astroblepus sp. & 7929 & 4228 & $\mathrm{C}$ \\
\hline Astroblepus cf unifasciatus & 7931 & & $\mathrm{C}$ \\
Astroblepus cf unifasciatus & 7934 & & $\mathrm{C}$ \\
Astroblepus cf unifasciatus & 7933 & \multirow{2}{*}{4229} & $\mathrm{C}$ \\
Astroblepus cf unifasciatus & 7930 & & $\mathrm{C}$ \\
Astroblepus cf unifasciatus & 7935 & & $\mathrm{C}$ \\
\hline Astrobus cf unifasciatus & 7932 & & $\mathrm{C}$ \\
\hline
\end{tabular}


Análisis filogenético de la secuencia del gen COI. Para la extracción de ADN se empleó el kit comercial GeneJet Genomic DNA Purification (Thermo Fisher Scientific). La amplificación fue realizada por PCR empleando los cebadores FishF1 (5'-TCAACCAACCACAAAGACATTGGCAC-3') y FishR1 (5'-TAGACTTCTGGGTGGCCAAAGAATCA-3') (Ward et al 2005). El volumen final de las reacciones de PCR fue $30 \mu 1$, conteniendo $19,88 \mu 1$ de agua ultrapura, 3,0 $\mu 1$ de Taq Buffer (10x), 4,80 $\mu \mathrm{L}$ de $\mathrm{MgCl}_{2}(25 \mathrm{mM})$, $0,48 \mu 1$ del mix de dNTPS $(2 \mathrm{mM}), 0,30 \mu 1$ de cada cebador $(10 \mathrm{mM}), 0,24 \mu 1 \mathrm{de}$ Taq Polimerasa y $1,0 \mu 1 \mathrm{de}$ ADN. El perfil térmico empleado para las amplificaciones fue: desnaturalización inicial por 2 minutos a $95^{\circ} \mathrm{C}$, seguido por 35 ciclos de desnaturalización a $94^{\circ} \mathrm{C}$ por 30 segundos, hibridación a $54{ }^{\circ} \mathrm{C}$ por 30 segundos y extensión a $72{ }^{\circ} \mathrm{C}$ por 1 minuto. Posteriormente se efectuó la extensión final a $72{ }^{\circ} \mathrm{C}$ por 10 minutos. Todos los productos de PCR fueron corroborados en gel de agarosa al $1 \%$ y posteriormente fueron secuenciados por el método estándar de Sanger en un secuenciador automatizado AB1 en Macrogen (Corea del Sur).

Las secuencias obtenidas se editaron y alinearon individualmente usando el programa Clustal W (Thomson et al. 1994) en el programa BIOEDIT 7. 0.5 (Hall 1999). Las secuencias consenso fueron alineadas y se construyó una matriz final, a partir de la cual se estimaron las distancias genéticas entre y dentro de los agrupamientos mediante el modelo de distancia de Kimura de dos parámetros (K2P), en el programa MEGA 5.01 (Tamura et al. 2011). Para los análisis de delimitación de especies, inicialmente estimamos un árbol ultramétrico usando el programa BEAST versión 1.7 (Drummond 2012), bajo el modelo de evolución $\mathrm{GTR}+\mathrm{G}+\mathrm{I}$. Los análisis se reañizaron con una longitud de 10.000.000 de cadenas MCMC (Cadena de Markov Monte Carlo), seguido por un período de burn-in del 10\%, alcanzando un nivel óptimo de convergencia entre el número de iteraciones y el logaritmo de la asignación probabilística. El árbol ultramétrico obtenido se empleó para realizar la delimitación de especies usando el Modelo "Generalized Mixed Yule Coalescent Model" (GMYC) en el programa R versión 3.0.0, usando el paquete "SpLiTS" (Species Limits by Threshold Statistics) y parámetros estándar (intervalo $=\mathrm{c}(0,10)$ ) que especifican el tiempo de transición entre y dentro de las especies. El uso del modelo GMYC se ha convertido en una aproximación efectiva para la delimitación de especies basadas en datos filogenéticos (Fujisawa y Barraclough 2013, Gerar et al. 2013, Lo et al. 2017). Este método no requiere información previa de las tasas de diversificación, y fue desarrollado específicamente para análisis usando un sólo locus y basado en la predicción que la evolución independiente conduce a la aparición de grupos genéticos distintos (Acinas et al. 2004, Barraclough y Herniou 2003).

Análisis corporal basado en proporciones y conteos. Con la finalidad de observar cuales caracteres morfológicos soportan las agrupaciones establecidas mediante GMYC, se tomaron 13 medidas del cuerpo de los 129 individuos (tabla 2). Se realizó el conteo de los radios de todas las aletas y la identificación del tipo de dientes tanto de la mandíbula inferior como de la premaxila. Las 13 medidas corporales fueron empleadas para realizar un análisis descriptivo de componentes principales, con la finalidad de encontrar "espacios de dimensiones más pequeñas" detectando qué o cuáles componentes explican la variabilidad entre los ejemplares muestreados; y un análisis discriminante para evaluar la relevancia de dichas variables en la conformación de grupos. Para realizar esto, las medidas se expresaron en razón de la longitud estándar (LE) como sugiere Wilson (1998), se transformaron a $\log _{10}$ y fueron probadas estadísticamente con Kruskall-Wallis aplicando el programa PAST (Hammer et al. 2001).

Tabla 2. Medidas lineales realizadas sobre el cuerpo de ejemplares del género Astroblepus

\begin{tabular}{lc}
\hline Medidas & $\begin{array}{c}\text { Abreviaturas } \\
\text { en el texto }\end{array}$ \\
\hline Longitud estándar & LE \\
\hline Longitud Total & LT \\
$\begin{array}{l}\text { Longitud de la cabeza } \\
\text { Distancia del extremo posterior de } \\
\text { la aleta dorsal a la base anterior del } \\
\text { pedúnculo caudal }\end{array}$ & DDBC \\
$\begin{array}{l}\text { Distancia de la base de la aleta } \\
\text { dorsal a la espina adiposa }\end{array}$ & DDPE \\
$\begin{array}{l}\text { Distancia de la espina adiposa al } \\
\text { pedúnculo caudal }\end{array}$ & DEPC \\
Longitud predorsal & LPD \\
Longitud prepélvica & LPPEL \\
Longitud preanal & LPA \\
$\begin{array}{l}\text { Distancia de la aleta pectoral a la } \\
\text { aletas pélvica }\end{array}$ & DPP \\
$\begin{array}{l}\text { Distancia de la aleta pélvica a la } \\
\text { aleta anal }\end{array}$ & DPA \\
\hline $\begin{array}{l}\text { Distancia Interorbital } \\
\text { Diámetro del ojo }\end{array}$ & DIO \\
\hline & DO \\
\hline
\end{tabular}




\section{RESULTADOS}

Caracterización genética. Se obtuvieron las secuencias del gen $C O I$ con una longitud de 580 pb para los 129 especímenes de Astroblepus. Las secuencias no presentaron inserciones, deleciones o codones de parada. La matriz presentó una composición nucleotídica de $25,7 \%$ adenina, $28,8 \%$ citosina, $16,6 \%$ guanina y $28,9 \%$ timina. Se observó un total de 36 haplotipos registrando una diversidad haplotípica $\mathrm{h}=0,664$. Considerando la matriz total, se identificaron 284 sitios conservados, 298 sitios polimórficos y 186 sitios informativos para parsimonia. Los análisis con el modelo GMYC indicaron que el umbral de tiempo antes del cual todos los nodos en el árbol reflejan eventos de diversificación y después del cual todos los nodos en el árbol reflejan eventos coalescentes es de $-0,006512577$. De acuerdo con lo anterior, el modelo identificó 12 entidades taxonómicas, soportado por un valor de verosimilitud para un modelo nulo de 1212,183 en comparación con la máxima probabilidad para el modelo GMYC de LM=1239,63 (figura 2). Las distancias genéticas K2P estimadas para los 12 agrupamientos (denotados con letras A-L) corroboran los resultados obtenidos del análisis de GMYC, con valores de 0,08 a 0,41 de distancia genética entre los agrupamientos, con un valor medio de 0,0219 (tabla 3). En comparación, las distancias intragrupos fueron bajas, con valores entre 0,001 y 0,004 .

\section{Descripción morfológica de los grupos identificados} genéticamente. Cada uno de los agrupamientos identificados en el análisis genético fue caracterizado morfológicamente usando los caracteres mencionados en la tabla 2 y tabla 4 . Los resultados del análisis estadístico de Kruskall-Wallis no mostraron diferencias significativas entre las variables morfológicas analizadas.

El grupo A presentó los dientes premaxilares incisivos y el perfil dorsal, a la altura del pedúnculo caudal, describió una línea recta. Los individuos incluidos en este grupo presentaron una longitud estándar entre 37,48-57,02 mm, longitud de la cabeza $0,276-0,324 \mathrm{~mm}$, distancia de la base posterior de la aleta dorsal a la base del radio no ramificado de la aleta caudal $0,42-0,46 \mathrm{~mm}$, distancia de la base de la aleta dorsal a la espina adiposa $0,363-0,402 \mathrm{~mm}$, distancia de la espina adiposa a la base del radio no ramificado de la aleta caudal $0,044-0,099 \mathrm{~mm}$, longitud predorsal $0,405-0,458$ $\mathrm{mm}$, longitud prepélvica $0,364-0,434 \mathrm{~mm}$, longitud preanal $0,702-0,809 \mathrm{~mm}$, distancia de las aletas pectorales a las pélvicas $0,134-0,246 \mathrm{~mm}$, distancia de la aleta pélvica a la aleta anal $0,317-0,429 \mathrm{~mm}$, distancia interorbital de 0,053-0,068 mm y diámetro del ojo de $0,02 \mathrm{~mm}$. Radios de la aleta dorsal: i, 6, pectoral: i, 11-12, anal: i, 6 .

El grupo B estuvo conformado por solo un individuo el cual presentó los dientes premaxilares incisivos y el perfil dorsal, a la altura del pedúnculo caudal, describió una línea curveada. Presentó una longitud estándar de 52,4 $\mathrm{mm}$, longitud de la cabeza $0,314 \mathrm{~mm}$, distancia de la base posterior de la aleta dorsal a la base del radio no ramificado de la aleta caudal $0,42 \mathrm{~mm}$, distancia de la base de la aleta

Tabla 3. Distancias genéticas interespecíficas obtenidas usando el modelo de Kimura 2 parámetros (K2P) para los 12 grupos de Astroblepus identificados con GYMC

\begin{tabular}{|c|c|c|c|c|c|c|c|c|c|c|c|}
\hline & A & B & C & D & E & $F$ & G & H & I & $\mathrm{J}$ & K \\
\hline B & 0,13 & & & & & & & & & & \\
\hline C & 0,09 & 0,08 & & & & & & & & & \\
\hline D & 0,22 & 0,18 & 0,14 & & & & & & & & \\
\hline$E$ & 0,15 & 0,17 & 0,13 & 0,27 & & & & & & & \\
\hline$F$ & 0,19 & 0,22 & 0,18 & 0,31 & 0,15 & & & & & & \\
\hline G & 0,16 & 0,19 & 0,15 & 0,28 & 0,11 & 0,083 & & & & & \\
\hline H & 0,17 & 0,19 & 0,16 & 0,28 & 0,10 & 0,114 & 0,081 & & & & \\
\hline I & 0,23 & 0,25 & 0,22 & 0,36 & 0,193 & 0,274 & 0,243 & 0,222 & & & \\
\hline J & 0,23 & 0,28 & 0,23 & 0,391 & 0,226 & 0,273 & 0,235 & 0,233 & 0,233 & & \\
\hline K & 0,26 & 0,287 & 0,25 & 0,398 & 0,249 & 0,298 & 0,252 & 0,265 & 0,238 & 0,248 & \\
\hline L & 0,25 & 0,39 & 0,26 & 0,41 & 0,25 & 0,30 & 0,257 & 0,256 & 0,265 & 0,29 & 0,274 \\
\hline
\end{tabular}




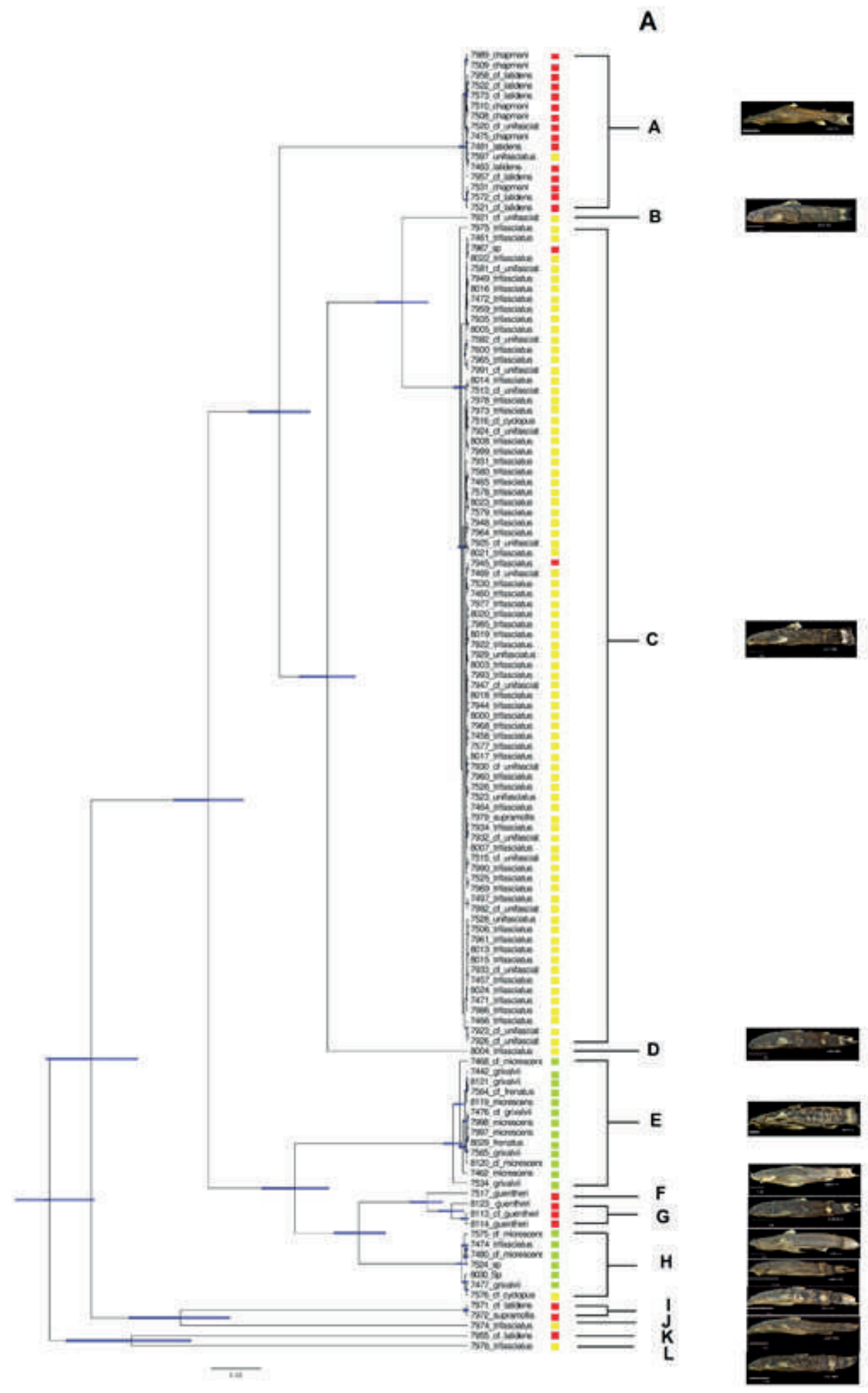

Figura 2: Árbol ultramétrico usando secuencias del gen Citocromo Oxidasa subunidad I (COI) para 129 especímenes del género Astroblepus. En la columna A se observan los 12 grupos obtenidos mediante MGYC. 


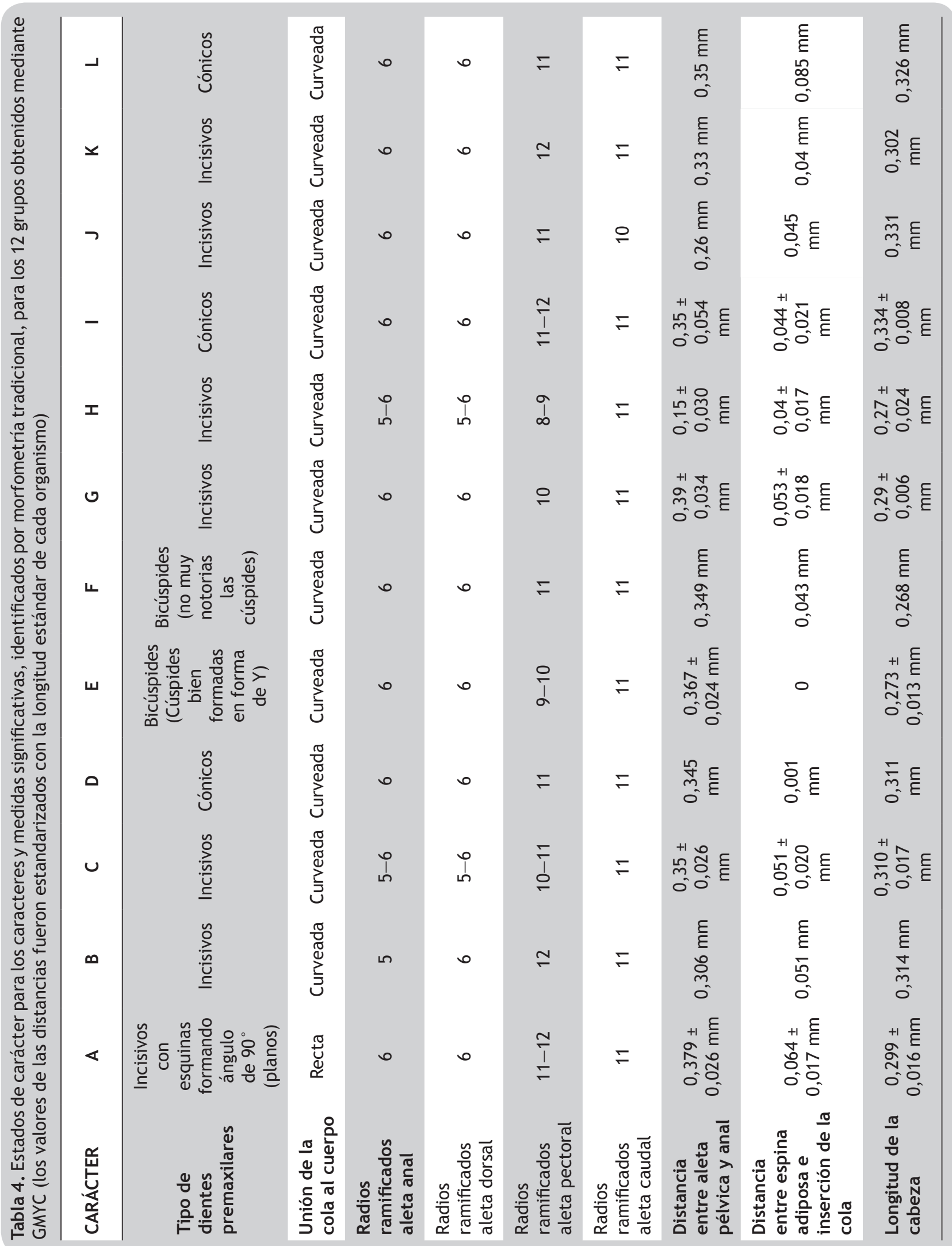


dorsal a la espina adiposa $0,418 \mathrm{~mm}$, distancia de la espina adiposa a la base del radio no ramificado de la aleta caudal $0,051 \mathrm{~mm}$, longitud predorsal $0,405 \mathrm{~mm}$, longitud prepélvica $0,306 \mathrm{~mm}$, longitud preanal $0,72 \mathrm{~mm}$, distancia de las aletas pectorales a las pélvicas $0,162 \mathrm{~mm}$, distancia de la aleta pélvica a la aleta anal $0,408 \mathrm{~mm}$, distancia interorbital de $0,057 \mathrm{~mm}$ y diámetro del ojo de $0,02 \mathrm{~mm}$. Radios de la aleta dorsal: i, 6, pectoral: i, 12, anal: i, 5 .

El grupo C, con el mayor número de individuos, se caracterizó por un radio blando no ramificado y 10-11 radios blandos ramificados en las aletas pectorales y radio blando no ramificado y 10-11 radios blandos ramificados en la aleta caudal. Los individuos presentaron una longitud estándar entre 21,57-63,37 mm, longitud de la cabeza $0,27-0,35 \mathrm{~mm}$, distancia de la base posterior de la aleta dorsal a la base del radio no ramificado de la aleta caudal $0,37-0,52 \mathrm{~mm}$, distancia de la base de la aleta dorsal a la espina adiposa $0,28-0,45 \mathrm{~mm}$, distancia de la espina adiposa a la base del radio no ramificado de la aleta caudal $0,023-0,131 \mathrm{~mm}$, longitud predorsal $0,42-0,50$ $\mathrm{mm}$, longitud prepélvica $0,19-0,79 \mathrm{~mm}$, longitud preanal 0,35-0,85 mm, distancia de las aletas pectorales a las pélvicas $0,11-0,24 \mathrm{~mm}$, distancia de la aleta pélvica a la aleta anal $0,23-0,35 \mathrm{~mm}$, distancia interorbital de 0,050-0,083 mm y diámetro del ojo de 0,019-0,04 mm. Radios de la aleta dorsal: i, 5-6, pectoral: i, 10-11, anal: i, 5-6 y caudal: ii, 10-11, y dientes de la premaxila incisivos.

El grupo D presentó la menor distancia entre la espina de la aleta adiposa y la base del radio no ramificado de la aleta caudal. Presentó una longitud estándar de 42,92 $\mathrm{mm}$, longitud de la cabeza $0,31 \mathrm{~mm}$, distancia de la base posterior de la aleta dorsal a la base del radio no ramificado de la aleta caudal $0,458 \mathrm{~mm}$, distancia de la base de la aleta dorsal a la espina adiposa $0,42 \mathrm{~mm}$, distancia de la espina adiposa a la base del radio no ramificado de la aleta caudal $0,001 \mathrm{~mm}$, longitud predorsal $0,45 \mathrm{~mm}$, longitud prepélvica $0,406 \mathrm{~mm}$, longitud preanal $0,77 \mathrm{~mm}$, distancia de las aletas pectorales a las pélvicas $0,20 \mathrm{~mm}$, distancia de la aleta pélvica a la aleta anal $0,35 \mathrm{~mm}$, distancia interorbital de $0,08 \mathrm{~mm}$ y diámetro del ojo de $0,027 \mathrm{~mm}$. Radios de la aleta dorsal: i, 6, pectoral: i, 11, anal: i, 6 y caudal: ii, 11 y los dientes de la premaxila cónicos.

El grupo E no presentó espina en la aleta adiposa y presentó un mayor diámetro del ojo. Los individuos presentaron longitud estándar entre 35,31-103,22 mm, longitud de la cabeza $0,26-0,30 \mathrm{~mm}$, distancia de la base posterior de la aleta dorsal a la base del radio no ramificado de la aleta caudal $0,44-0,51 \mathrm{~mm}$, distancia de la base de la aleta dorsal a la espina adiposa $0,48-0,56 \mathrm{~mm}$, distancia de la espina adiposa a la base del radio no ramificado de la aleta caudal $0,02-0,05 \mathrm{~mm}$, longitud predorsal $0,37-0,43 \mathrm{~mm}$, longitud prepélvica $0,36-0,42 \mathrm{~mm}$, longitud preanal $0,73-0,79 \mathrm{~mm}$, distancia de las aletas pectorales a las pélvicas $0,18-0,22$ $\mathrm{mm}$, distancia de la aleta pélvica a la aleta anal $0,32-0,40$ $\mathrm{mm}$, distancia interorbital de 0,05-0,07 mm y diámetro del ojo de 0,021-0,039 mm. Radios de la aleta dorsal: i, 6, pectoral: i, 9-10, anal: i, 6 y caudal: ii, 11 y los dientes de la premaxila bicúspides.

El grupo F presentó los dientes de la premaxila bífidos y puntiagudos. Los individuos pertenecientes a este grupo presentaron una longitud estándar entre 35,31-128,4 mm, longitud de la cabeza $0,269-0,294 \mathrm{~mm}$, distancia de la base posterior de la aleta dorsal a la base del radio no ramificado de la aleta caudal $0,44-0,55 \mathrm{~mm}$, distancia de la base de la aleta dorsal a la espina adiposa $0,48-0,58 \mathrm{~mm}$, longitud predorsal $0,36-0,42 \mathrm{~mm}$, longitud prepélvica $0,36-0,42 \mathrm{~mm}$, longitud preanal $0,73-0,79 \mathrm{~mm}$, distancia de las aletas pectorales a las pélvicas $0,19-0,22 \mathrm{~mm}$, distancia de la aleta pélvica a la aleta anal $0,32-0,42 \mathrm{~mm}$, distancia interorbital de $0,056-0,078$ $\mathrm{mm}$ y diámetro del ojo de 0,02-0,05 mm (lucen "ojones" con respecto a los otros individuos examinados). Radios de la aleta dorsal: i, 6, pectoral: i, 9-10, anal: i, 6 y caudal: ii, 11 y dientes de la premaxila bicúspides.

El grupo G presentó la mayor separación entre las aletas pélvicas y anal y los dientes premaxilares fueron bicúspides pero redondeados; la longitud estándar entre 55,8-62,2 $\mathrm{mm}$, longitud de la cabeza $0,287-0,299 \mathrm{~mm}$, distancia de la base posterior de la aleta dorsal a la base del radio no ramificado de la aleta caudal $0,40-0,46 \mathrm{~mm}$, distancia de la base de la aleta dorsal a la espina adiposa $0,37-0,39$ $\mathrm{mm}$, distancia de la espina adiposa a la base del radio no ramificado de la aleta caudal $0,034-0,069 \mathrm{~mm}$, longitud predorsal $0,44-0,453 \mathrm{~mm}$, longitud prepélvica $0,37-0,43$ $\mathrm{mm}$, longitud preanal $0,75-0,85 \mathrm{~mm}$, distancia de las aletas pectorales a las pélvicas $0,19-0,22 \mathrm{~mm}$, distancia de la aleta pélvica a la aleta anal $0,36-0,43 \mathrm{~mm}$, distancia interorbital de 0,054-0,067 mm y diámetro del ojo de 0,02 $\mathrm{mm}$. Radios de la aleta dorsal: i, 6 , pectoral: i, 10, anal: i, 6 , dientes de la premaxila incisivos y dientes maxilares en forma de corona no muy pronunciados.

El grupo H presentó una cabeza pequeña y las aletas dorsal y caudal presentaron un radio blando no ramificado y 5-6 radios blandos ramificados. Los individuos pertenecientes a este grupo presentaron una longitud estándar entre 45,38-53,58 $\mathrm{mm}$, longitud de la cabeza $0,256-0,268 \mathrm{~mm}$, distancia de la base posterior de la aleta dorsal a la base del radio no 
ramificado de la aleta caudal $0,49-0,51 \mathrm{~mm}$, distancia de la base de la aleta dorsal a la espina adiposa $0,42-0,48 \mathrm{~mm}$, distancia de la espina adiposa a la base del radio no ramificado de la aleta caudal $0,022-0,071 \mathrm{~mm}$, longitud predorsal $0,35-0,38 \mathrm{~mm}$, longitud prepélvica $0,27-0,42 \mathrm{~mm}$, longitud preanal $0,371-0,798 \mathrm{~mm}$, distancia de las aletas pectorales a las pélvicas $0,187-0,212 \mathrm{~mm}$, distancia de la aleta pélvica a la aleta anal 0,316-0,362 $\mathrm{mm}$, distancia interorbital de 0,053-0,064 mm y diámetro del ojo de 0,02-0,03 mm. Radios de la aleta dorsal: i, 5-6, pectoral: i, 8-11, anal: i, 5-6, y dientes de la premaxila incisivos.

El grupo I presentó la cabeza más grande del grupo de los especímenes analizados. Los individuos pertenecientes a este grupo presentaron una longitud estándar entre 26,49-28,3 mm, longitud de la cabeza 0,33-0,34 mm, distancia de la base posterior de la aleta dorsal a la base del radio no ramificado de la aleta caudal 0,37-0,39 mm, distancia de la base de la aleta dorsal a la espina adiposa 0,31-0,36 mm, distancia de la espina adiposa a la base del radio no ramificado de la aleta caudal $0,05-0,03 \mathrm{~mm}$, longitud predorsal $0,45 \mathrm{~mm}$, longitud prepélvica $0,44-0,45$ $\mathrm{mm}$, longitud preanal $0,74-0,80 \mathrm{~mm}$, distancia de las aletas pectorales a las pélvicas $0,16-0,19 \mathrm{~mm}$, distancia de la aleta pélvica a la aleta anal $0,31-0,39 \mathrm{~mm}$, distancia interorbital de 0,06-0,08 mm y diámetro del ojo de 0,02-0,04 mm. Radios de la aleta dorsal: i, 6, pectoral: i, 11-12, anal: i, 6 , y dientes de la premaxila cónicos.

El grupo J presentó la menor separación entre las aletas pélvicas y anales. La longitud estándar fue $37,96 \mathrm{~mm}$, longitud de la cabeza $0,33 \mathrm{~mm}$, distancia de la base posterior de la aleta dorsal a la base del radio no ramificado de la aleta caudal $0,44 \mathrm{~mm}$, distancia de la base de la aleta dorsal a la espina adiposa $0,39 \mathrm{~mm}$, distancia de la espina adiposa a la base del radio no ramificado de la aleta caudal $0,045 \mathrm{~mm}$, longitud predorsal $0,47 \mathrm{~mm}$, longitud prepélvica $0,44 \mathrm{~mm}$, longitud preanal $0,70 \mathrm{~mm}$, distancia de las aletas pectorales a las pélvicas $0,21 \mathrm{~mm}$, distancia de la aleta pélvica a la aleta anal $0,26 \mathrm{~mm}$, distancia interorbital de $0,07 \mathrm{~mm}$ y diámetro del ojo de $0,03 \mathrm{~mm}$. Radios de la aleta dorsal: i, 6, pectoral: i, 11, anal: i, 6, y dientes de la premaxila incisivos.

El grupo K presentó los dientes de la premaxila incisivos y la aleta pectoral presentó 12 radios blandos ramificados; una longitud estándar de 46,24 mm, longitud de la cabeza $0,30 \mathrm{~mm}$, distancia de la base posterior de la aleta dorsal a la base del radio no ramificado de la aleta caudal $0,41 \mathrm{~mm}$, distancia de la base de la aleta dorsal a la espina adiposa $0,41 \mathrm{~mm}$, distancia de la espina adiposa a la base del radio no ramificado de la aleta caudal $0,039 \mathrm{~mm}$, longitud predorsal 0,42 mm, longitud prepélvica $0,43 \mathrm{~mm}$, longitud preanal $0,75 \mathrm{~mm}$, distancia de las aletas pectorales a las pélvicas $0,19 \mathrm{~mm}$, distancia de la aleta pélvica a la aleta anal $0,33 \mathrm{~mm}$, distancia interorbital de $0,06 \mathrm{~mm}$ y diámetro del ojo de 0,02 mm. Radios de la aleta dorsal: i, 6, anal: i, 6 .

Finalmente, el grupo L presentó una mayor distancia de la espina adiposa a la base del radio no ramificado dorsal de la aleta caudal, una longitud estándar $36,44 \mathrm{~mm}$, longitud de la cabeza $0,33 \mathrm{~mm}$, distancia de la base posterior de la aleta dorsal a la base del radio no ramificado de la aleta caudal 0,45 mm, distancia de la base de la aleta dorsal a la espina adiposa $0,36 \mathrm{~mm}$, distancia de la espina adiposa a la base del radio no ramificado de la aleta caudal $0,09 \mathrm{~mm}$, longitud predorsal $0,47 \mathrm{~mm}$, longitud prepélvica $0,39 \mathrm{~mm}$, longitud preanal $0,70 \mathrm{~mm}$, distancia de las aletas pectorales a las pélvicas $0,15 \mathrm{~mm}$, distancia de la aleta pélvica a la aleta anal 0,35 mm, distancia interorbital de 0,06 $\mathrm{mm}$ y diámetro del ojo de $0,03 \mathrm{~mm}$. Radios de la aleta dorsal: i, 6, anal: i, 6 y los dientes de la premaxila son incisivos.

De acuerdo con los grupos identificados y la agrupación de dos o más individuos, se realizó una clave dicotómica para facilitar la identificación en campo. Todas las medidas fueron estandarizadas respecto la longitud estándar.

1a. Longitud de la cabeza cabe 3,72 a 3,9 veces en LE GRUPO H.

1b. Longitud de la cabeza cabe 4 veces o más en LE 2

2a. Línea dorsal a la altura del pedúnculo caudal describe una línea recta (figura 3A-B)

GRUPO A.

2b. Línea dorsal a la altura del pedúnculo caudal describe un ángulo

3a. Aleta adiposa presente, extendiéndose desde la base posterior de la aleta dorsal hasta la base del radio no ramificado de la aleta caudal, sin espina adiposa presente o evidente. (figura 3C-D)

GRUPO E.

3b. Aleta adiposa presente, reducida o moderada. Espina adiposa presente y evidente

4

4a. Distancia entre la aleta pélvica y la aleta anal cabe de 2,3 a 2,74 veces en LE

GRUPO G.

4b. Distancia entre la aleta pélvica y la aleta anal cabe de 2,79 a 3,4 veces en $\mathrm{LE}$ GRUPO C. 


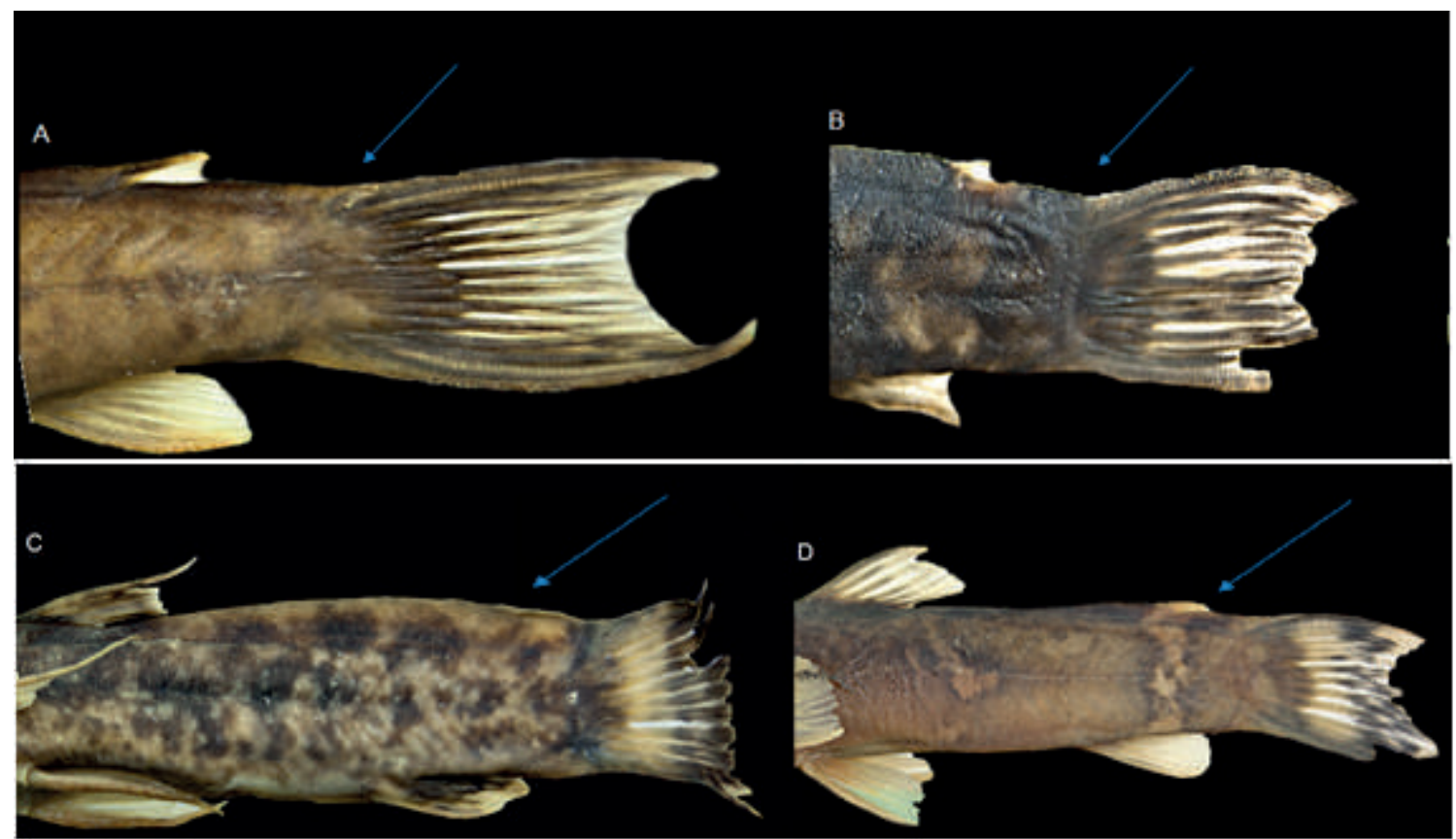

Figura 3: Caracteres diagnósticos para los especímenes de Astroblepus. A. Inserción recta de la cola a la base del radio simple de la aleta caudal. B. Inserción ondulada u oblicua de la cola a la base del radio simple de la aleta caudal. C. Aleta adiposa bien desarrollada sin espina adiposa evidente. D. Aleta adiposa moderada con espina adiposa evidente.

Para cada uno de los grupos determinados anteriormente, y debido a que no se contaba con revisión de ejemplares tipo, ni ejemplares de diversas localidades, ni un número representativo de ejemplares por grupo, no se asignó un epíteto específico existente, pero se realizó una identificación a nivel de grupo, considerando las características de los individuos pertenecientes a él, según las claves taxonómicas hasta hoy disponibles, denominándolos así: Grupo A (grupo latidens), Grupo C (grupo trifasciatus), Grupo E (grupo grixalvii), Grupo G (grupo guentheri), Grupo H (grupo micrescens).

\section{DISCUSIÓN}

Los análisis moleculares empleando la región del gen COI han sido utilizados, durante la última década, para la identificación genética de especies y su eficacia se ha validado en varios grupos de animales (Waugh 2007), incluidos los peces (Rosso et al. 2012, Valdez et al. 2009, Ward et al. 2005). Ward et al. (2005) proporcionaron pruebas de la eficacia del ADN "barcoding" en la identificación de peces marinos, en un estudio que examinó más de 200 especies australianas. En general, la identificación de especies a través del código de barras de ADN constituye una poderosa herramienta para la identificación de la diversidad de especies, principalmente en grupos donde es limitada la identificación de caracteres morfológicos únicos, relacionada principalmente con su alta variación y sobreposición (Camacho et al. 2011, Talavera et al. 2013, Tapan et al. 2017). A nivel molecular, la información genética de los individuos revela la variación que ha sido fijada en el genoma y que ha permitido su diversificación.

Una de las principales dificultades en la identificación de especies del género Astroblepus se debe a que la mayoría de sus especies son definidas por un único carácter o por combinaciones de caracteres externos que muestran altos niveles de variación inter e intraespecífica (Schaefer 2011). Claramente, esto puede ser observado al comparar la identificación inicial de los individuos analizados en estudio y los agrupamientos definidos por el análisis de delimitación de especies, siendo que, muchos de los especímenes que conformaban un mismo lote fueron asignados en diferentes grupos. Igualmente, análisis morfométricos previos para el mismo conjunto de individuos identificaron únicamente tres grupos (Hernández et al. 2016). Como se evidencia en revisiones taxonómicas realizadas para el género, muchos de los caracteres usados para definir los límites entre especies 
de Astroblepus, como la forma del cuerpo, el tamaño del cuerpo y la configuración, así como los patrones de conformación, han sido confundidos con variación a diferentes niveles, que pueden resultar de complejas contribuciones de múltiples fuentes tanto intrínsecas como extrínsecas, tales como la ontogenia, el dimorfismo sexual y la variación geográfica (Miranda-Chumacero 2006), lo que dificulta la delimitación de especies por métodos morfológicos, tal como fue observado durante este estudio. No obstante, considerando la importancia de la identificación morfológica, es indispensable la realización de una revisión taxonómica completa para las especies del género en Colombia, empleando tanto herramientas moleculares, así como explorando otro tipo de caracteres morfológicos (osteológicos, miológicos y merísticos) que permitan una adecuada delimitación de las especies.

De acuerdo con los resultados obtenidos, la diversidad de especies ha sido sobre-estimada y hasta el momento, para el área de estudio, pueden identificarse claramente 12 unidades taxonómicas operacionales (OTU's), las cuales son soportadas por los valores de distancia genética. A pesar que los límites de diferenciación basados en la distancia genética dependen del conocimiento de las relaciones filogenéticas de un grupo taxonómico, considerando la ausencia de este tipo de información para el grupo de estudio, nuestros análisis de diferenciación genética siguen a Ward (2009), el cual sugiere que valores interespecíficos mayores a $3 \%$ y valores intraespecíficos menores al $1 \%$ pueden ser límites usados para la identificación de especies; si un espécimen desconocido tiene más de $2 \%$ de divergencia, con un nivel de confianza mayor al 95\%, puede ser considerado como una especie diferente. No obstante, considerando la escasa información disponible para el grupo, estos resultados deben ser sujetos a posterior corroboración. Actualmente no existe un banco de datos para especies del género distribuidas en Colombia incluido en el Fishbold y solo se han registrado secuencias en el GenBank para especies distribuidas en Perú (Schaefer et al. 2011) lo que limita un análisis comparativo de los resultados obtenidos.

Aunque no se determinaron características únicas que permitieran diferenciar los grupos establecidos, la descripción morfológica de las OTU's identificadas en este estudio representa una contribución importante en la identificación de los especímenes de las quebradas afluentes al río Porce.

En conclusión, nuestros resultados resaltan la importancia del uso de herramientas moleculares y morfológicas en la identificación de especies que presentan altos niveles de variación intraespecífica.

\section{AGRADECIMIENTOS}

A las personas que colaboraron en la recolecta de los individuos de Astroblepus en el monitoreo del río Porce: Alejandro Loaiza, Andrés Hernández, Daniel Santamaría, Diego Cataño, Jonathan Álvarez, Juan Diego Correa y Juan Pablo Londoño; a Jonathan Álvarez por la dedicación, compañía y asesoría en la toma de fotografías de los ejemplares, al profesor Juan Manuel Daza por su apoyo y acompañamiento y a la Universidad de Antioquia por la financiación del proyecto.

\section{REFERENCIAS}

Acinas SG, Klepac-Ceraj V, Hunt DE, Pharino C, Ceraj I, Distel DL, Polz MF. 2004. Fine-scale phylogenetic architecture of a complex bacterial community. Nature, 430(6999): 551-554. DOI:10.1038/nature02649

Barraclough TG, Herniou E. 2003. Why do species exist? Insights from sexuals and asexuals, Zoology (Jena), 106(4): 275-282. DOI:10.1078/0944-2006-00130.

Boulenger, GA. 1887. On new siluroid fishes from the Andes of Columbia. Annals and magazine of natural history, 19(113): 348350. https://doi.org/10.1080/00222938709460255.

DrummondA, Suchard M, Xie D, RambautA. 2012. Bayesian phylogenetics with BEAUti and the BEAST 1.7., Molecular Biology and Evolution, 29(8): 1969-1973. doi:10.1093/molbev/mss075

Empresas Públicas de Medellín E.S.P. 2004. Análisis físico-químicos de las aguas del embalse Porce II años 2001-2003, Área Hidrometría e Instrumentación. Medellín (Colombia): Empresas Públicas de Medellín.

Eigenmann CH. 1912. Some results from an ichthyological reconnaissance of Colombia, South America. Part II. Bloomington (USA): Indiana University studies, 16(8): 1-27. http://www.scielo.org.co/scielo. php?script=sci_arttext\&pid=S0123-30682013000200009.

Eigenmann CH. 1918. Eighteen new species of fishes from northwestern South America. Proceedings of the American Philosophical Society, 56(7): 673-689.

Eschmeyer WNR, Fong JD. 2017. Catalog of fishes. San Francisco (CA): California Academy of Sciences; [fecha de acceso mayo 1, 2017]. http://researcharchive.calacademy.org/research/ichthyology/ catalog/speciesbyfamily.asp

Fujisawa T, Barraclough TG. 2013. Delimiting species using singlelocus data and the generalized mixed Yule coalescent approach: a revised method and evaluation on simulated data sets. Systematic Biology, 62(5): 707-24. doi:10.1093/sysbio/syt033

Gerard T, Vlad D, Vila R.2013. Factors affecting species delimitation with the GMYC model: insight from a butterfly survey. Methods in Ecology and Evolution, 4(12): 1101-1110. https://doi. org/10.1111/2041-210X.12107. 
Hall T. 1999. BioEdit: a user-friendly biological sequence alignment editor and analysis program for Windows 95/98/NT. Nucleic Acids Symposium, 41: 95-98.

Hammer Ø, Harper DAT, Ryan PD. 2001 PAST: Paleontological statistics software package for education and data analysis. Paleontologia electronica, [fecha de acceso noviembre 20, 2016]; 4(1): 9. https://palaeo-electronica.org/2001_1/past/past.pdf

Hebert PD, Cywinska A, Ball SL, deWaard JR. 2003. Biological identifications through DNA barcodes. Biological Science, 270(1512): 313-321. DOI: 10.1098/rspb.2002.2218.

Hernández M, Ochoa L, Jiménez LF. 2016. Integración de métodos de DNA barcode y morfometría geométrica revelan diversidad de especies del género Astroblepus en la cuenca del río Porce, Colombia [Trabajo de pregrado]. [Medellín (Colombia)]: Instituto de Biología, Universidad de Antioquia.

Humboldt F. 1805. Mémoire sur une nouvelle espèce de pimelode, jetée par les volcans du Royaume de Quito. En: Voyage de Humboldt et Bonpland, Deuxième partie. Observations de Zoologie et d'Anatomie comparée. Paris: F. Schoell. 3(7): 21-25.

Jacobsen D. 2008. Tropical high-altitude streams. En: D Dudgeon editor. Tropical Stream Ecology. Netherlands: Elsevier. p. 219-256.

Jiménez LF, Álvarez J, Ochoa LE, Loaiza A, Londoño JP, Restrepo D, Aguirre K, Hernández A, Correa JD, Jaramillo U. 2014. Guía Ilustrada Peces Cañón del río Porce, Antioquia. Medellín (Colombia): Empresas Públicas de Medellín.

Johnson RD. 1912. Notes on the habits of a climbing catfish (Arges marmoratus) from the Republic of Colombia. Annals of the New York Academy of Sciences, [fecha de acceso junio 15, 2016]; 22(1): 327-333. https://nyaspubs.onlinelibrary.wiley.com/doi/ pdf/10.1111/j.1749-6632.1912.tb55167.x

Lo PC, Liu SH, Nor SAM, Chen WJ. 2017. Molecular exploration of hidden diversity in the Indo-West Pacific sciaenid clade. PloS one, [fecha de acceso mayo 29, 2017]; 12(7). DOI:10.1371/ journal. pone. 0176623 .

Miles C. 1971. Los peces del río Magdalena. ("A field book of Magdalena fishes”). 2a edición. Ibagué (Colombia): Universidad del Tolima ediciones.

Miranda-Chumacero G. 2006. Distribución altitudinal, abundancia relativa y densidad de peces en el río Huarinilla y sus tributarios (Cotapata, Bolivia). Ecología en Bolivia, [fecha de acceso marzo 15, 2016]; 41(1): 79-93. http://www.scielo.org.bo/scielo. php?script=sci_arttext\&pid=S1605-25282006000700006\&lng $=$ es\&nrm=iso

Pearson N. 1937. The fishes of the Atlantic and Pacific slopes near Cajamarca, Peru. Proceedings of the California Academy of Sciences, 23(4): 87-98. https://www.biodiversitylibrary.org/ page/3188251\#page/113/mode/1up

Regan CT. 1904. A monography of the family Loricariidae. Transactions of the Zoological Society of London, 17(3): 191-350. https://doi. org/10.1111/j.1096-3642.1904.tb00040.x

Reis E, Sven O, Ferraris C, editores. 2003. Check List of the Freshwater Fishes of South and Central America. Porto Alegre (Brasil): Edipucrs.
Rosso J, Mabragaña E, González C, Astarloa D. 2012. DNA barcoding Neotropical fishes: recent advances from the Pampa Plain, Argentina. Molecular Ecology Resources. [fecha de acceso febrero 15, 2016]; 12(6): 999-1011. https://doi.org/10.1111/1755-0998.12010.

Schaefer S, Arroyave J. 2010. Rivers as islands: determinants of the distribution of Andean astroblepid catfishes. Journal of Fish Biology, [fecha de acceso mayo 21, 2015]; 77(10). DOI: 10.1111/j.1095-8649.2010.02822.x.

Schaefer S, Chakrabarty P, Geneva A, Sabaj M. 2011. Nucleotide sequence data confirm diagnosis and local endemism of variable morphospecies of andean astroblepid catfishes (siluriformes: Astroblepidae). Zoological Journal of the Linnean Society, 162(1): 90-102. https://doi.org/10.1111/j.1096-3642.2010.00673.x

Steindachner F. 1882. Beiträge zur Kenntniss der Flussfische Südamerika's (IV). Anzeiger der Akademie für Wissenschaftlichen, Wien 19(19): 175-180.

Talavera G, Dinca V, Vila R. 2013. Factors affecting species delimitations with the GMYC model: insight from a butterfly survey. Methods in Ecology and Evolution, 4(12): 1101-1110. https://doi.org/10.1111/2041-210X.12107

Tapan KB, Surya NS, Bijayalaxmi S, Bibarani T, Usha R. 2017 Morphological and genetic analyses of the first record of longrakered trevally, Ulua mentalis (Perciformes: Carangidae) and of the pinjalo snapper, Pinjalo pinjalo (Perciformes: Lutjanidae) in the Odisha coast, Bay of Bengal. Mitochondrial DNA Part A, DNA mapping, sequencing and analysis, 29(4): 552560. https://doi.org/10.1080/24701394.2017.1320993

Tamura K, Peterson D, Peterson N, Stecher G, Nei M, Kumar S. 2011. MEGA5: molecular evolutionary genetics analysis using maximum likelihood, evolutionary distance, and maximum parsimony methods. Molecular Biology and Evolution, 28(10): 2731-2739. DOI: $10.1093 / \mathrm{molbev} / \mathrm{msr} 121$

Thompson JD, Higgins DG, Gibson TJ. 1994. CLUSTAL W: improving the sensitivity of progressive multiple sequence alignment through sequence weighting, position-specific gap penalties and weight matrix choice. Nucleic Acids Research, 22(22): 4876-4880.

Valdez M, Ivanova V, Gutiérrez M, Contreras S, Hebert PD. 2009. Probing diversity in freshwater fishes from Mexico and Guatemala with DNA barcodes. Journal of Fish Biology, 74(2): 377-402. https://doi.org/10.1111/j.1095-8649.2008.02077.x

Ward RD, Zemlak TS, Innes BH, Last PR, Hebert PD. 2005. DNA barcoding Australia's fish species. Philosophical Transactions of the Royal Society B, 360(1462): 1847- 1857. DOI:10.1098/ rstb.2005.1716

Ward RD. 2009. DNA barcode divergence among species and genera of birds and fishes. Molecular Ecology Resource, 9(4): 1077-1085. DOI: 10.1111/j.1755-0998.2009.02541.x

Waugh J. 2007. DNA barcoding in animal species: progress, potential and pitfalls. BioEssays, 29(2): 188-197. DOI: 10.1002/bies.20529

Wilson G. 1998. A description of the early juvenile colour patterns of eleven Lethrinus species (Pisces: Lethrinidae) from the Great Barrier Reef, Australia. Records of the Australian Museum, 50(1): 55-83. DOI:10.3853/j.0067-1975.50.1998.1274 